Original Article

\title{
A CROSS-SECTIONAL STUDY ON MENTAL HEALTH AND CARDIOVASCULAR REACTIVITY AMONG FRESH RESIDENT DOCTORS DURING COVID19 PANDEMIC IN INDIA
}

\author{
SUMIT KUMAR ${ }^{1}$, ARIJITA BANERJEE ${ }^{* 1}$, AQUIL AHMAD ${ }^{2}$ \\ ${ }^{1}$ Institute of Human Behaviour and Allied Sciences, Delhi, ${ }^{2 *}$ Maulana Azad Medical College, Delhi \\ Email: b.arijita@gmail.com
}

Received: 20 Jan 2021, Revised and Accepted: 25 Mar 2021

\section{ABSTRACT}

Objective: The adverse psychological impact in doctors battling the Corona Virus Disease 2019 (COVID 19) epidemic arise from various social and administrative issues like excessive workload, inadequate interpersonal communications, biased work culture and lack of social support thus raising a warning of the mental health in doctors. The present study was done to assess the mental health and stress reactivity among the resident doctors who have been working for a prolonged duration during covid 19 pandemic in India.

Methods: The subjects included 162 young resident doctors both males and females, of age group between 25-30 y, among which 92 were posted in covid wards on a rotation basis from $1^{\text {st }}$ April 2020 to $31^{\text {st }}$ December 2020 and 70 were doing duty in regular non-covid wards or not had done even a single duty in covid ward during the above period. All participants completed an online questionnaire that included the Beck Anxiety Inventory (BAI), the Beck Depression Inventory (BDI)-II, Connor-Davidson resilience scale (CDRISC) and Social Support Rating Scale (SSRS) followed by a cold pressor test to assess stress reactivity.

Results: There were significant differences in the mental health and stress reactivity scores with lower resilience scores, lower social support rating scores, increased anxiety and depression in Covid ward doctors $(\mathrm{p}=0.001)$.

Conclusion: The findings suggested high levels of training, resilience helpful social support and unbiased work culture were necessary to health care workers engaged in public health emergence.

Keywords: Resilience, Anxiety, Cardiovascular reactivity

(C) 2021 The Authors. Published by Innovare Academic Sciences Pvt Ltd. This is an open access article under the CC BY license (https://creativecommons.org/licenses/by/4.0/) DOI: https://dx.doi.org/10.22159/ijcpr.2021v13i3.42094 Journal homepage: https://innovareacademics.in/journals/index.php/ijcpr

\section{INTRODUCTION}

Corona virus disease, originating in Wuhan, in December 2019, has lead to immense suffering to more than 200 countries worldwide. Among all substantial economic, social and psychological impacts, healthcare systems have been drastically affected making the healthcare workers vulnerable to stress, anxiety, loneliness, depression, emotional exhaustion and feelings of helplessness. Since, COVID19 pandemic is public health emergency of international concern, the healthcare workers irrespective of experience, are bound to put maximum possible efforts in mitigating the spread and decreasing the mortality from COVID 19 disease [1].

Those who have newly joined the public health emergency as resident doctors or those having no previous experience of any healthcare emergency are confronted to more stress and the capability to deal with such adversity that is one's resilience, has become a matter of concern now. Yet the adverse psychological impact in them arise from various social and administrative issues like excessive workload, inadequate personal protective equipments, exaggerating media news, inadequate and inappropriate social support thus raising a warning towards increasing rates of suicides and alcohol abuse among healthcare workers. A recent study by Lai et al. depicted the above psychological burden among the frontline workers and nurses in Wuhan during late January 2020. Similar study by Wang et al. reported poor sleep, depression and anxiety among the people in China during the crisis $[2,3]$.

Various intercontinental surveys having conducted to report regarding the mental health status of the frontline workers, but the present study was done to assess the mental health and cardiovascular reactivity among the resident doctors working in the capital city of India, Delhi.

\section{MATERIALS AND METHODS}

\section{Methods}

A cross-sectional survey of young resident doctors was approved by the Institutional Ethics Committee. The subjects included 162 young resident doctors both males and females, of age group between 25$30 \mathrm{y}$, among which 92 were posted in covid wards on a rotation basis from $1^{\text {st }}$ April 2020 to $31^{\text {st }}$ December 2020 and 70 were doing duty in regular non-covid wards or not had done even a single duty in covid ward during the above period. The above resident doctors posted in covid wards had no previous exposure to any kind of public health emergency. The subjects with any comorbid conditions like hypertension, diabetes mellitus, bronchial asthma, hypothyroidism, hyperthyroidism, any renal disease, any endocrine disorder etc, or under any medication or with a habit of smoking or tobacco chewing, all are excluded from the present study.

The informed consent was received from all the subjects, after collecting basic information about name, age, gender and duration with nature of duty, the subjects were asked to fill out the questionaire forms. The link of the questionnaire was mailed to all the subjects individually.

In the present study, all participants completed an online questionnaire that included the Beck Anxiety Inventory (BAI), the Beck Depression Inventory (BDI)-II, Connor-Davidson resilience scale (CDRISC) and Social Support Rating Scale (SSRS). Following which Resting blood pressure and heart rate (baseline) were measured at ambient temperature using a mercury sphygmomanometer. Each subject was exposed to cold pressor test [4-9].

The subject was asked to immerse his/her hand in ice-cold water 4 ${ }^{\circ} \mathrm{C}$ for 1 min or till he perceives the pain. At the end of 1 and $5 \mathrm{~min}$ heart rate and blood pressure measurements are to be taken Reactivity score $(\triangle \mathrm{CPT})$ are calculated. ${ }^{9}$ Cardiovascular parameters measured following CPT were:-

Blood pressure-Systolic Blood Pressure (SBP) and Diastolic Blood Pressure (DBP) were measured by using mercury sphygmomanometer.

Pulse rate was measured clinically using the three-finger method on the radial artery. 
Pulse pressure was calculated by subtracting SBP with DBP (SBPDBP).

Mean arterial pressure was calculated by the formula MAP= DBP+1/3PP.

Rate pressure product (RPP) was calculated by using formula $\mathrm{RPP}=(\mathrm{SBP} * \mathrm{PR}) / 100$.

The Beck Anxiety Inventory (BAI) is an inventory with 21 items measuring both somatic and cognitive symptoms of anxiety. It has a likert scale ranging from 0 to 3 while its absolute scores range from 0 to 63 . The reliability coefficient is 0.92 and test-retest reliability is 0.75. It is known for its good discriminative validity and internal consistency. However, the BAI focuses much on the psychophysiological symptoms related to panic [4].

The original Beck Depression Inventory (BDI) was developed in 1961. Since then several versions had been put forward and finally BDI-II was developed to correspond to DSM-IV criteria for diagnosing depression thus including items pertaining to cognition, affection, somatic and vegetative symptoms of depression. There are 21 items in BDI-II with a 4 point likert scale indicating the degree of severity of depression ranging from 0 (not at all) to 3 (extreme form of one symptom). Total score being 63 , the more high the score, the more severe is the depression. The cronbach alpha was reported to be 0.92 for BDI-II and it has got improved content validity by adding items to assess various somatic and cognitive criteria of depression with high clinical usability and research usability $[5,6]$.

The CD-RISC is a measure of stress coping ability in a person. It determines how one is able to bounce back after stressful or adverse circumstances in life. it helps a person to stay focussed and think clearly while not getting discouraged by failure. it consists of 25 items each rated on a 5 point scale $(0-4)$. Total possible scores range from 0 to 100 . The test is known for adequate internal consistency, test-retest reliability and validity [7]
Social support is defined as the physical or psychological help that people receive through social connections which in turn can reduce stress, decrease tension, improve quality of life and adaptation capabilities. It is an annual and protection given to each other. SSRS consists of 10 items pertaining to 3 dimensions of social support, subjective support (4 items), objective (3 items) and support-seeking behaviour (3 items). Subjective support counts on the interpersonal network of an individual. Objective support measures the extent of support an individual has obtained in his past. Support seeking behaviour refers to the approach a person make while asking for social support. Total scores add up to 66, higher the scores indicate stronger social support. SSRS is having adequate reliability and validity [8].

Demographic backgrounds of doctors were computed. The di fferences of mental health, resilience, social support and stress reactivity between covid ward duty doctors and non-covid ward duty doctors were compared via simple independent sample t-test. $\mathrm{P}<0.05$ was considered statistically signi ficant. SPSS 20.0 was used to conduct the analysis.

\section{RESULTS}

The demographic factors shown in table 1, were comparable between the two groups. In table 2, mental health differences between covid ward doctors and noncovid ward doctors were compared. We found statistically significant differences in both anxiety and depression scales between two groups $(\mathrm{p}=.001)$. Further when we evaluated resilience in table 3 , using CD-RISC we observed significant lower scores in terms of strength and optimism in covid ward doctors $(\mathrm{p}=0.001)$. In table 4 , social support rating scores were significantly less in covid ward doctors in terms of objective support and subjective support while there was no significant differences in availability of support between groups.

Table 5 shows the result of stress reactivity to cold pressor test where all cardiovascular parameters, Pulse rate, Mean Arterial pressure and Rate pressure product, showed significant differences between groups $(\mathrm{p}=0.001)$.

Table 1: Distribution of samples

\begin{tabular}{llll}
\hline Demographics & Groups & COVID ward doctors (92) & Non-COVID ward doctors (70) \\
\hline gender & male & $49(30.24 \%)$ & $38(23.45 \%)$ \\
& females & $43(26.5 \%)$ & $32(19.75 \%)$ \\
Marital status & married & $56(34.5 \%)$ & $44(27.16 \%)$ \\
& unmarried & $32(19.75 \%)$ & $26(16.04 \%)$ \\
\hline
\end{tabular}

Table 2: Mental health of COVID ward doctors and non-COVID ward doctors

\begin{tabular}{llll}
\hline Scales & COVID ward doctors & Non-COVID ward doctors & p-value \\
\hline Anxiety & $48.74 \pm 3.02$ & $43.57 \pm 2.68$ & 0.001 \\
Depression & $32.64 \pm 0.35$ & $31.34 \pm 0.29$ & 0.003 \\
\hline
\end{tabular}

Table 3: Resilience of COVID ward doctors and non-COVID ward doctors

\begin{tabular}{llll}
\hline Scales & COVID ward doctors & Non-COVID ward doctors & p-value \\
\hline Tenacity & $34.62 \pm 1.04$ & $35.08 \pm 0.32$ & 0.32 \\
Strength & $21.44 \pm 3.92$ & $26.72 \pm 2.76$ & $<0.001$ \\
Optimism & $8.78 \pm 1.36$ & $10.98 \pm 2.49$ & $<0.001$ \\
CD-RISC & $64.84 \pm 6.33$ & $72.78 \pm 5.57$ & $<0.001$ \\
\hline
\end{tabular}

Table 4: Social support of COVID ward doctors and non-COVID ward doctors

\begin{tabular}{llll}
\hline Scales & COVID ward doctors & Non-COVID ward doctors & p-value \\
\hline Objective support & $9.46 \pm 2.80$ & $10.30 \pm 2.76$ & 0.05 \\
Subjective support & $23.82 \pm 4.92$ & $25.88 \pm 2.34$ & 0.002 \\
Availability & $8.12 \pm 2.68$ & $8.34 \pm 1.92$ & 0.56 \\
SSRS & $41.4 \pm 7.34$ & $44.52 \pm 6.92$ & 0.006 \\
\hline
\end{tabular}


Table 5: Stress reactivity of COVID ward doctors and non-COVID ward doctors

\begin{tabular}{llll}
\hline Delta change score & COVID ward doctors & Non-COVID ward doctors & p-value \\
\hline PR & $2.16 \pm 0.72$ & $1.34 \pm 1.2$ & 0.001 \\
MAP & $22.54 \pm 1.19$ & $21.92 \pm 0.84$ & 0.003 \\
RPP & $20.52 \pm 2.73$ & $18.84 \pm 1.64$ & 0.001 \\
\hline
\end{tabular}

\section{DISCUSSION}

Several studies depicted the association of social support, interpersonal relationships, work environment to mental health in an individual. Doctors who have been working in the frontline during covid 19 since more than a year are in high risk group to develop anxiety and depression. The current study emphasized that lack of personal coping strategies, individual vulnerability, reduced resilience, limited social support, lack of training and experience could aggravate such stress reactions $[10,11]$.

During Covid 19 pandemic, resident doctors had to isolate themselves in one room to prevent cross-infection and enhance social distancing, which caused tremendous level of fear, anxiety and despair among them due to lack of social support and interpersonal communication [12].

Another interesting point to ponder upon from our study is the lack of support from peers. Resilience is supposed to be the protective factor to mental health. Yet the resilience scores were significantly lower in covid ward doctors compared to noncovid doctors. Certainly good social support from colleagues and administration leading to unbiased work distribution would have buffered the stress among the covid ward doctors, especially for the fresh staff [13].

At rest, the sympathetic nervous system does not seem to be activated, but a greater cardiovascular reactivity to physical stress like cold pressor test had been documented in covid ward doctors in our study. Various studies previously have shown a strong association between increased cardiovascular reactivity and future hypertension $[14,15]$.

\section{LIMITATIONS}

Sleep quality of the doctors and their quality of life in isolation facilities were not taken into account, which could have been another potential factor to mental health.

\section{CONCLUSION}

Our study concluded that doctors working for prolonged duration during covid 19 pandemic showed worse performance in mental health, resilience, social support and are at risk for future cardiovascular diseases due to exaggerated stress reactivity.

Therefore, it is important to have a good level of training in healthcare workers, helpful peer support, unbiased work environment, continuous screening facilities to evaluate the mental health and potential risk factors of healthcare workers during public health emergency.

\section{ACKNOWLEDGEMENT}

This research received no specific grant from any funding agency in the public, commercial, or not-for-profit sectors.

\section{CONFLICTS OF INTERESTS}

The Authors declare that there is no conflict of interest.

\section{REFERENCES}

1. Banerjee D. The COVID-19 outbreak: crucial role the psychiatrists can play. Asian J Psychiatry 2020;50:102014. https://doi.org/10.1016/j.ajp.2020.102014.

2. Allen J, Balfour R, Bell R, Marmot M. Social determinants of mental health. Int Rev Psychiatry 2014;26:392-407.

3. Barkur G, Vibha Kamath GB. Sentiment analysis of nationwide lockdown due to COVID 19 outbreak: evidence from India. Asian J Psychiatry 2020;51:102089.

4. Beck AT, Ward CH, Mendelson M, Mock J, Erbaugh J. An inventory for measuring depression. Arch Gen Psychiatry 1961;4:561-71.

5. Dar KA, Iqbal $\mathrm{N}$, Mushtaq A. Intolerance of uncertainty, depression, and anxiety: examining the indirect and moderating effects of worry. Asian J Psychiatr 2017;29:129-33.

6. Xiao H, Zhang Y, Kong D, Li S, Yang N. The effects of social support on sleep quality of medical staff treating patients with coronavirus disease 2019 (COVID-19) in January and February 2020 in China. Med Sci Monitoring 2020;26:e923549. https://doi.org/10.12659/MSM.923549

7. Connor K, Davidson J. Development of a new resilience scale: the connor davidson resilience scale (CD-RISC). Depress Anxiety 2003;18:76-82.

8. Hamaideh HS. Burnout, social support, and job satisfaction among Jordanian mental health nurses. Issues Ment Health Nurs 2011;32:234-42.

9. Stewart KM, France CR. Resting systolic blood pressure, parental history of hypertension, and sensitivity to noxious stimuli. Pain 1996;68:369-74.

10. Xiang YT, Yang Y, Li W, Zhang $Q$, Cheung T, Hg CH. Timely mental health care for the 2019 novel coronavirus outbreak is urgently needed. Lancet Psychiatry 2020;7:228-9.

11. Leary AO, Jalloh MF, Neria Y. Fear and culture: contextualizing mental health impact of the 2014-2016 Ebola epidemic in West Africa. Br Med J Global Health 2018;3:e000924. https://doi.org/10.1136/bmjgh-2018000924

12. Tsang HW, Scudds RJ, Chan EY. Psychosocial impact of SARS (Letter). Emerging Infect Dis 2004;10:1326-7.

13. Brooks SK, Webster RK, Smith LE, Woodland L, Wessely S, Greenberg $\mathrm{N}$, et al. The psychological impact of quarantine and how to reduce it: rapid review of the evidence. Lancet 2020;395:912-20.

14. Wang C, Horby PW, Hayden FG, Gao GF. A novel coronavirus outbreak of global health concern. Lancet 2020;395:470-3.

15. A Banerjee, M Bedi, VP Varshney. Cardiovascular reactivity to mental and physical stress in offspring of hypertensive parents. National J Physiol Pharm Pharmacol 2021;11:134-7. 